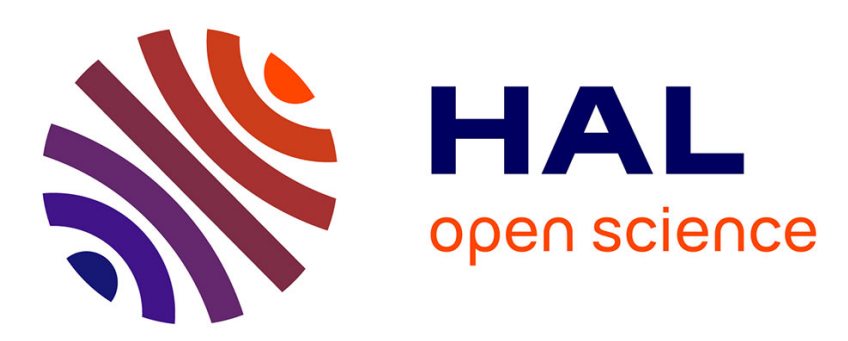

\title{
Unifying different interpretations of the nonlinear response in glass-forming liquids
}

P. Gadige, S. Albert, M. Michl, Th Bauer, P. Lunkenheimer, A. Loidl, R. Tourbot, C. Wiertel-Gasquet, G. Biroli, J.-P. Bouchaud, et al.

\section{- To cite this version:}

P. Gadige, S. Albert, M. Michl, Th Bauer, P. Lunkenheimer, et al.. Unifying different interpretations of the nonlinear response in glass-forming liquids. Physical Review E , 2017, 96, pp.32611. 10.1103/PhysRevE.96.032611 . cea-01634913

\section{HAL Id: cea-01634913 https://hal-cea.archives-ouvertes.fr/cea-01634913}

Submitted on 14 Nov 2017

HAL is a multi-disciplinary open access archive for the deposit and dissemination of scientific research documents, whether they are published or not. The documents may come from teaching and research institutions in France or abroad, or from public or private research centers.
L'archive ouverte pluridisciplinaire HAL, est destinée au dépôt et à la diffusion de documents scientifiques de niveau recherche, publiés ou non, émanant des établissements d'enseignement et de recherche français ou étrangers, des laboratoires publics ou privés. 


\title{
Unifying different interpretations of the nonlinear response in glass-forming liquids
}

\author{
P. Gadige,,${ }^{1, *}$ S. Albert,${ }^{1, \dagger}$ M. Michl, ${ }^{2}$ Th. Bauer, ${ }^{2, \dagger}$ P. Lunkenheimer, ${ }^{2}$ A. Loidl,${ }^{2}$ R. Tourbot,${ }^{1}$ C. Wiertel-Gasquet,${ }^{1}$ \\ G. Biroli, ${ }^{3,4}$ J.-P. Bouchaud, ${ }^{5}$ and F. Ladieu ${ }^{1, \S}$ \\ ${ }^{1}$ SPEC, CEA, CNRS, Université Paris-Saclay, CEA Saclay, Bâtiment 772, 91191 Gif-sur-Yvette Cedex, France \\ ${ }^{2}$ Experimental Physics V, Center for Electronic Correlations and Magnetism, University of Augsburg, 86159 Augsburg, Germany \\ ${ }^{3} I P h T, C E A, C N R S$, Université Paris-Saclay, CEA Saclay, Bâtiment 774, 91191 Gif-sur-Yvette Cedex, France \\ ${ }^{4}$ LPS, Ecole Normale Supérieure, 24 Rue Lhomond, 75231 Paris Cedex 05, France \\ ${ }^{5}$ Capital Fund Management, 23 Rue de l'Université, 75007 Paris, France
}

(Received 25 February 2017; revised manuscript received 9 June 2017; published 27 September 2017)

\begin{abstract}
This work aims at reconsidering several interpretations coexisting in the recent literature concerning nonlinear susceptibilities in supercooled liquids. We present experimental results on glycerol and propylene carbonate, showing that the three independent cubic susceptibilities have very similar frequency and temperature dependences, for both their amplitudes and phases. This strongly suggests a unique physical mechanism responsible for the growth of these nonlinear susceptibilities. We show that the framework proposed by two of us [J.-P. Bouchaud and G. Biroli, Phys. Rev. B 72, 064204 (2005)], where the growth of nonlinear susceptibilities is intimately related to the growth of glassy domains, accounts for all the salient experimental features. We then review several complementary and/or alternative models and show that the notion of cooperatively rearranging glassy domains is a key (implicit or explicit) ingredient to all of them. This paves the way for future experiments, which should deepen our understanding of glasses.
\end{abstract}

DOI: 10.1103/PhysRevE.96.032611

\section{INTRODUCTION}

Glassy materials represent a very wide class of everyday materials ranging from molecular glasses to granular systems and from polymers to colloids and foams. Yet the microscopic mechanisms leading to the spectacular increase of their relaxation time with temperature or density are still controversial. In particular, the existence of an underlying thermodynamic critical point, which would explain why rigidity develops in these systems, is a hotly debated issue [1].

In the past 15 years, however, some consensus has emerged about the existence of a growing length scale accompanying the slowing down of the dynamics of these various materials. Although anticipated by Adam and Gibbs [2] more than 50 years ago, the status of this length scale has remained elusive for a long time. For example, it was often argued that within the mode-coupling theory of glasses the dynamical arrest phenomena are purely local [3]. However, quite the contrary was shown in [4-6]. The random first-order transition (RFOT) theory provides a consistent framework in which to understand the intuition of Adam and Gibbs: A supercooled liquid should be thought of as a mosaic of locally rigid but amorphous regions, the size of which increases as the temperature is reduced [7]. The necessity of a growing length scale in super-Arrhenius systems, an argument put forward

\footnotetext{
*Present address: Complex Fluids Lab, Soft Condensed Matter Group, Raman Research Institute, CV Raman Avenue, Sadashivanagar, Bangalore 560080, India.

†Present address: Laboratoire de Physique, Ecole Normale Supérieure de Lyon, 46 Avenue d'Italie, 69364 Lyon Cedex 07, France.

${ }^{\ddagger}$ Present address: Institute for Machine Tools and Industrial Management, Technical University of Munich, 85748 Garching, Germany.

${ }^{\S}$ Corresponding author: francois.ladieu@cea.fr
}

by many, was finally proved by Montanari and Semerjian in [8]. These theoretical breakthroughs have spurred a flurry of experimental and numerical attempts to elicit this length scale, directly or indirectly (see, e.g., [9]).

Among the different investigation tools, nonlinear effects are especially interesting: The nonlinear susceptibility is expected to have very different behavior when a genuine amorphous order sets in, as within RFOT theory, in contrast to the case of purely dynamical scenarios, such as that provided by kinetically constrained models (KCMs) [10], for which nontrivial thermodynamic correlations are absent. In particular, based on an analogy with spin glasses where the third-order static susceptibility $\chi_{3}$ is known to diverge at the transition, two of us [Bouchaud and Biroli (BB)] proposed in [11] that the nonlinear ac susceptibility of glasses should peak at a frequency of the order of the inverse relaxation time, with a peak height that increases as the number $N_{\text {corr }}$ of molecules collectively involved in typical relaxation events. In the spirit of the fluctuation-response theorem, the increase of the peak of $\chi_{3}$ reveals the growth of quasistatic amorphous correlations in the system [see Eqs. (4) and (7) below].

The predictions of BB have been broadly confirmed using nonlinear dielectric response in several experimental setups, first in glycerol [12], then in several other glass formers [13] and in plastic crystals [14], as well as for various pressures [15]. In all these studies, the temperature behavior of $N_{\text {corr }}$ (inferred from the peak of $\chi_{3}$ ) is in reasonable agreement with other, more indirect evidence [13-17]. These experiments have recently been extended to the fifth-order nonlinear susceptibility $\chi_{5}$ in glycerol and propylene carbonate [18] and are again fully consistent with BB's picture. In fact, the growth of the peak of $\chi_{5}$ as the temperature is reduced is stronger than that of $\chi_{3}$ and provides strong qualitative and quantitative evidence for the existence of an underlying critical point that drives the physics of supercooled liquids [18]. We also note that the nonlinear mechanical response has also been studied 
in colloids $[19,20]$, extending BB's results $[11,21]$ to that case as well.

However, alternative theoretical interpretations have recently been proposed [22,23], invoking other effects to explain the nonlinear effects, seemingly unrelated to the growth of $N_{\text {corr }}$. In order to clarify this issue, in the present paper we present additional experimental observations (Sec. II). We show that all three independent cubic ac susceptibilities have very similar frequency and temperature dependences, and their phases are related to one another. As we will show (Sec. III), this is very natural if the physical origin is the same and due to the increase of $N_{\text {corr }}$, but it is instead at odds with simple phenomenological pictures. Furthermore, we show (Sec. IV) that some of the alternative arguments can only explain the experimental results if some cooperative effects are present, as assumed by BB.

\section{THREE KINDS OF $\chi_{3}$ AND THEIR EMPIRICAL BEHAVIOR}

\section{A. Setup and definitions}

We first recall the general formalism defining the third-order susceptibilities by introducing the time-dependent kernel $\chi_{3}$, relating polarization and electric field as follows:

$$
\begin{aligned}
\frac{P(t)-P_{\text {lin }}(t)}{\epsilon_{0}}= & \iiint \chi_{3}\left(t-t_{1}^{\prime}, t-t_{2}^{\prime}, t-t_{3}^{\prime}\right) \\
& \times E\left(t_{1}^{\prime}\right) E\left(t_{2}^{\prime}\right) E\left(t_{3}^{\prime}\right) d t_{1}^{\prime} d t_{2}^{\prime} d t_{3}^{\prime}+\cdots,
\end{aligned}
$$

where higher-order terms in the field are not written because they correspond to higher-order susceptibilities and where $\epsilon_{0}$ is the vacuum dielectric constant. Note that the threefold convolution product contained in Eq. (1) is a simple generalization of the standard onefold convolution product used to express the linear response. In purely ac experiments where the magnitude of the oscillating field $E_{\text {ac }}$ (of angular frequency $\omega=2 \pi f$ ) is varied, two cubic responses arise, at frequencies $\omega$ and $3 \omega$. If a static field $E_{\mathrm{st}}$ is superimposed on top of $E_{\mathrm{ac}}$, new cubic responses arise, for both even and odd harmonics. By setting $\delta P \equiv P\left(E_{\mathrm{ac}}, E_{\mathrm{st}}\right)-P_{\mathrm{lin}}\left(E_{\mathrm{ac}}, E_{\mathrm{st}}\right)$ and keeping only the odd harmonics, we get

$$
\begin{aligned}
\frac{\delta P}{\epsilon_{0}}= & \frac{3}{4}\left|\chi_{3}^{(1)}\right| E_{\mathrm{ac}}^{3} \cos \left(\omega t-\delta_{3}^{(1)}\right) \\
& +\frac{1}{4}\left|\chi_{3}^{(3)}\right| E_{\mathrm{ac}}^{3} \cos \left(3 \omega t-\delta_{3}^{(3)}\right) \\
& +3\left|\chi_{2,1}^{(1)}\right| E_{\mathrm{st}}^{2} E_{\mathrm{ac}} \cos \left(\omega t-\delta_{2,1}^{(1)}\right),
\end{aligned}
$$

where we have used the threefold Fourier transform of the kernel introduced in Eq. (1) and defined

$$
\begin{aligned}
& \left|\chi_{3}^{(1)}\right| \exp \left(-i \delta_{3}^{(1)}\right):=\chi_{3}(-\omega, \omega, \omega), \\
& \left|\chi_{3}^{(3)}\right| \exp \left(-i \delta_{3}^{(3)}\right):=\chi_{3}(\omega, \omega, \omega), \\
& \left|\chi_{2,1}^{(1)}\right| \exp \left(-i \delta_{2,1}^{(1)}\right):=\chi_{3}(0,0, \omega) .
\end{aligned}
$$

For any cubic susceptibility, generically denoted by $\chi_{3}$, the corresponding dimensionless cubic susceptibility $X_{3}$ is defined as

$$
X_{3}=\frac{k_{B} T}{\epsilon_{0} \Delta \chi_{1}^{2} a^{3}} \chi_{3},
$$

where $\Delta \chi_{1}$ is the dielectric strength, i.e., $\Delta \chi_{1}=$ $\chi_{\operatorname{lin}}(0)-\chi_{\operatorname{lin}}(\infty)$, where $\chi_{\operatorname{lin}}(0)$ and $\chi_{\operatorname{lin}}(\infty)$ are, respectively, the linear susceptibility at zero and infinite frequency. Note that $X_{3}$ has the great advantage of being both dimensionless and independent of the field amplitude. Similar quantities can be defined for dimensionless fifth-order responses, as explained in Ref. [18].

Considering Eq. (1), one anticipates theoretically that the three cubic susceptibilities are closely related, since they all originate from the same pulse response function. However, it was claimed in Refs. [22,23] that the several unrelated effects contributing to the three $X_{3}$ 's could be singled out by a separate measurement of each cubic susceptibility. In this work we unveil the deep similarities existing between $X_{3}^{(1)}, X_{3}^{(3)}$, and $X_{2,1}^{(1)}$ that we have experimentally determined in glycerol and propylene carbonate, which are archetypical glass formers. The experiments were done in the Augsburg and Saclay setups described elsewhere [13,18,24]. For each data point of Figs. 1 and 2, the field was varied to ensure that the data obey Eq. (2); for the specific case of $X_{2 ; 1}^{(1)}$ the ac field $E_{\text {ac }}$ was kept well below the static field $E_{\mathrm{st}}$. We briefly emphasize that the nonlinear effects reported here have been shown to be free of exogeneous effects: The global homogeneous heating of the samples by the dielectric energy dissipated by the application of the strong ac field $E_{\text {ac }}$ was shown to be fully negligible for $X_{3}^{(3)}$ as long as the inverse of the relaxation time $f_{\alpha} \leqslant 1 \mathrm{kHz}$ (see Ref. [25]). These homogeneous heating effects were kept negligible in $X_{3}^{(1)}$ (to which they contribute much more) by keeping $f_{\alpha}$ below $10 \mathrm{~Hz}$ for the Saclay setup [24] or by severely limiting the number $n$ of periods during which the electric field is applied (Augsburg setup; see [26]). The contribution of electrostriction was demonstrated to be safely negligible in Ref. [24], both using theoretical estimates and by showing that changing the geometry of spacers does not affect $X_{3}^{(3)}$. As for the $\sim 0.5 \%$ ionic impurities present in both liquids, we briefly explain that they have a negligible role, except at zero frequency where it might explain why the three $X_{3}$ 's are not strictly equal, contrarily to what is expected on general grounds. Let us recall that on the one hand it was shown that ion heating contribution is fully negligible in $X_{2,1}^{(1)}$ (see the Appendix of Ref. [27]) and, on the other hand, it is well known that ions affect the linear response $\chi_{\text {lin }}$ at very low frequencies (say, $f / f_{\alpha} \lesssim 0.05$ ): This yields an upturn of the out-of-phase linear response $\chi_{\text {lin }}^{\prime \prime}$, which diverges as $1 / \omega$ instead of vanishing as $\omega$ in an ideally pure liquid containing only molecular dipoles. This is why we do not push our nonlinear measurements below $0.01 f_{\alpha}$, because at lower frequencies the nonlinear response is likely to be dominated by the ion contribution. In the same spirit, when measuring $X_{2 ; 1}^{(1)}$, the static field was applied during a finite amount of time (longer than $1 / \omega$ ) and its direction was systematically reversed to minimize any ionic migration effect. Finally, to avoid mixing the cubic response of molecular dipoles with that of ions, we have not measured the cubic response obtained just by using a pure static field. Therefore, we do not reach the zero-frequency limit where, on general grounds, one expects all the cubic susceptibilities to be equal. We think this is the reason why in Figs. 1 and 2 the three cubic susceptibilities are still slightly different even at the lowest frequencies that we have investigated. 

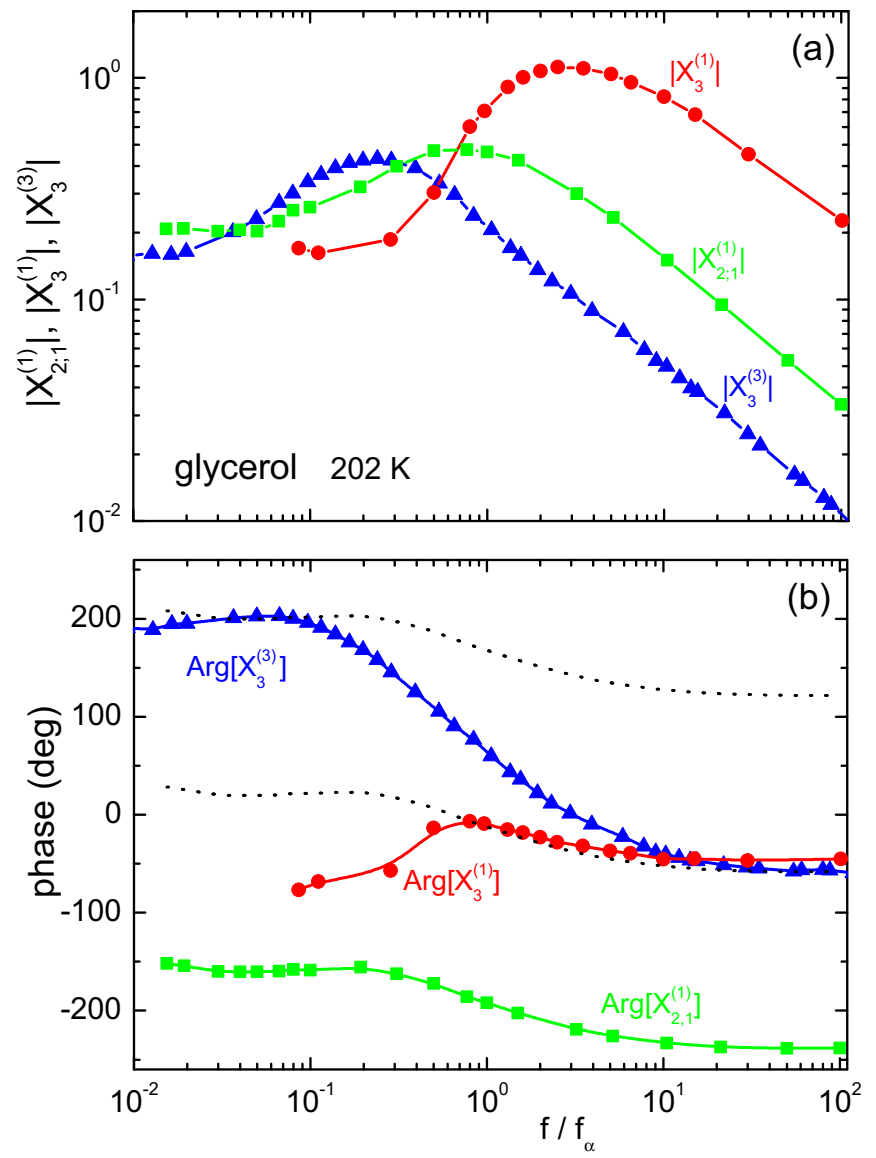

FIG. 1. Comparison of three cubic dimensionless susceptibilities of glycerol recorded at the same temperature with the same samples. The field amplitude is varied in $[2 \mathrm{MV} / \mathrm{m} ; 5 \mathrm{MV} / \mathrm{m}]$ (Saclay setup [24]). (a) Amplitudes and (b) phases are shown by the symbols; lines are just a guide for the eyes. Here $X_{3}^{(1)}$ and $X_{3}^{(3)}$ correspond to pure ac experiments of the first- and third-harmonic cubic response, respectively, and $X_{2,1}^{(1)}$ is the cubic response at the first harmonic when a dc field is superimposed on an ac field. Note the strong similarities between these three quantities. Moreover, the temperature behavior of the peak is the same for the three dimensionless susceptibilities reported here. The two dotted lines in (b) correspond to a global shift of $\operatorname{Arg}\left[X_{2,1}^{(1)}\right]$ either by $180^{\circ}$ [supporting Eq. (5)] or by $360^{\circ}$ (see the text).

\section{B. Experimental results}

Figure 1 shows the behavior of the three cubic susceptibilities for supercooled glycerol at $202 \mathrm{~K}$ where the inverse relaxation time $f_{\alpha} \simeq 2 \mathrm{~Hz}$. Figure 2 reports the same results for propylene carbonate at $160 \mathrm{~K}$ where $f_{\alpha} \simeq 0.2 \mathrm{~Hz}$. Figures 1 (a) and 2(a) display the moduli of the cubic susceptibilities, while Figs. 1(b) and 2(b) show the associated phases. We find four salient experimental features.

(i) The three moduli have a humped shape in frequency, with a peak located in the region of $f_{\alpha}$, namely, at $0.22 f_{\alpha}$ for $X_{3}^{(3)}$, at $0.8 f_{\alpha}$ for $X_{2,1}^{(1)}$, and at $2.5 f_{\alpha}$ for $X_{3}^{(1)}$. These three numerical prefactors are only slightly different in propylene carbonate. Above the peak (higher frequencies), the moduli of the three cubic susceptibilities decrease as $\sim\left(f_{\alpha} / f\right)^{0.6}$ for glycerol and as $\sim\left(f_{\alpha} / f\right)^{0.7}$ for propylene carbonate. Below the peak (lower
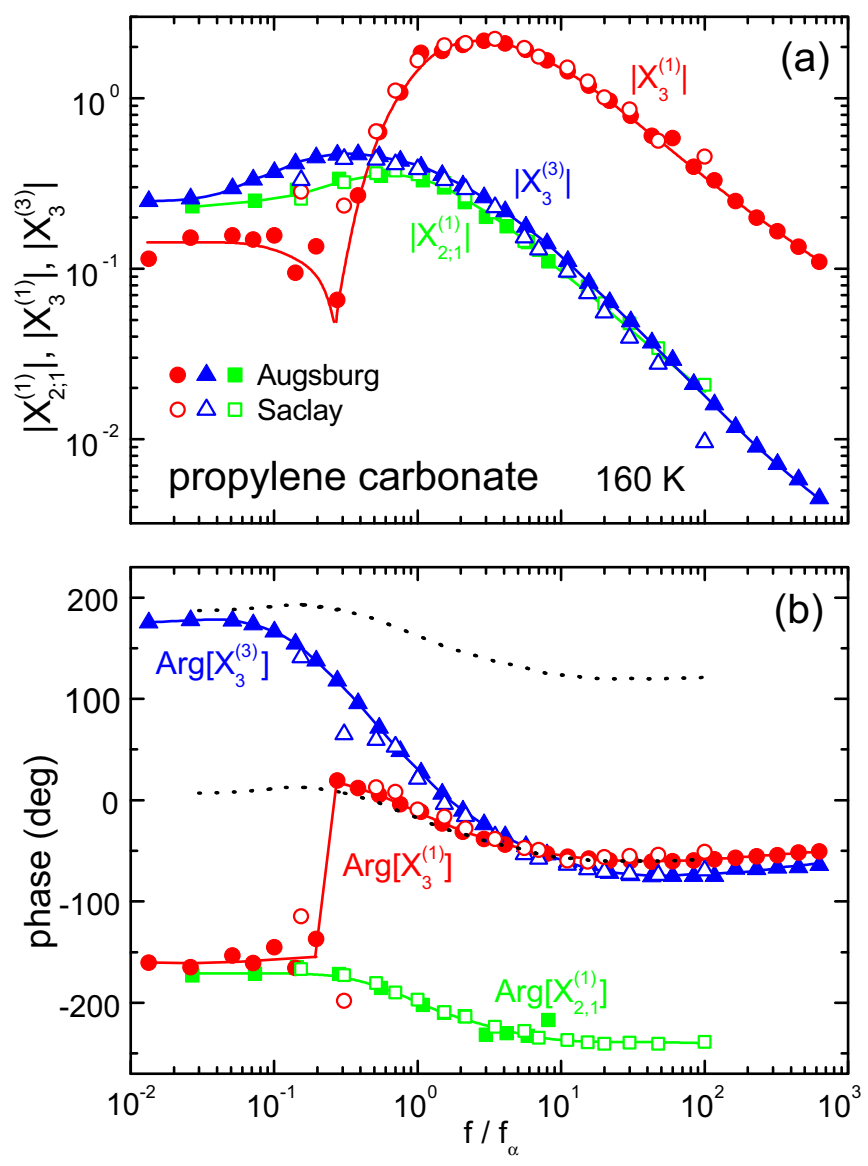

FIG. 2. Same as Fig. 1 but for propylene carbonate (lines are a guide for the eyes). The field amplitude is varied in $[2 \mathrm{MV} / \mathrm{m}$; $5 \mathrm{MV} / \mathrm{m}$ ] in the Saclay setup [24] and in [2.6 MV/m;3.5 MV/m] in the Augsburg setup [13].

frequencies), the moduli fall down when decreasing frequency and become independent of frequency when $f / f_{\alpha} \leqslant 0.05$. We refer to this low-frequency domain as the plateau region [28].

(ii) The temperature dependence of the three dimensionless susceptibilities is significantly stronger around and above the hump than in the plateau region. Around and above the hump, the three cubic susceptibilities have a temperature dependence that is very close to that of $T \chi_{T}:=T\left|\partial \ln f_{\alpha} / \partial T\right|$ (see Refs. [12,13,15,24,27]). Note that, owing to the limited temperature interval accessible to experiments, one cannot distinguish clearly between $T \chi_{T}$ and $T^{2} \chi_{T}$ (see Refs. [13,14,29]). As for the plateau region, its temperature dependence is much weaker. It was convincingly shown in Refs. [12,24,27] that for glycerol, $X_{3}^{(3)}$ and $X_{2,1}^{(1)}$ do not depend on temperature in the plateau region, up to the experimental accuracy of $\pm 3 \%$ per data point. This is also the case for propylene carbonate [13] where the plateau region lies in the same range of $f / f_{\alpha}$. Finally, the measurements of $X_{3}^{(3)}$ at various pressures was achieved in Ref. [15] and it was shown that the effect of pressure can be related to the effect of temperature.

(iii) The phases of the three cubic responses basically do not depend explicitly on temperature [12,24], but only on $u=f / f_{\alpha}$, through a master curve that depends only on the precise cubic susceptibility under consideration (see Figs. 1 
and 2). These master curves have the same qualitative shape as a function of $u$ in both glycerol and propylene carbonate. We note that the phases of the three cubic susceptibilities are related to one another. In the plateau region all the phases are equal (see the upper dotted line in Figs. 1 and 2), which is easily understood because at low frequency the system responds adiabatically to the external field. At higher frequencies, we note that for glycerol (expressing the phases in radians)

$$
\begin{gathered}
\operatorname{Arg}\left[X_{3}^{(1)}\right] \approx \operatorname{Arg}\left[X_{2,1}^{(1)}\right]+\pi \text { for } f / f_{\alpha} \geqslant 0.5, \\
\operatorname{Arg}\left[X_{3}^{(1)}\right] \approx \operatorname{Arg}\left[X_{3}^{(3)}\right] \quad \text { for } f / f_{\alpha} \geqslant 5,
\end{gathered}
$$

which are quite nontrivial relations, which hold also for propylene carbonate.

(iv) In the phase of $\chi_{3}^{(1)}$ of propylene carbonate (Fig. 2), a jump of $\pi$ is observed that is accompanied by the indication of a spikelike minimum in the modulus (more details are given in the Appendix, Sec. 1). A similar jump may also be present in glycerol (Fig. 1). We observe that this jump in the phase happens at the crossover between the $T$-independent plateau and the strongly $T$-dependent hump. More precisely, in the plateau region one observes a reduction of the real part of the dielectric constant $\chi_{\text {lin }}^{\prime}$, while around the hump $\chi_{\text {lin }}^{\prime}$ is enhanced. At the frequency of the jump, both effects compensate and this coincides with a very low value of the imaginary part of $X_{3}^{(1)}$.

Apart from this jump of $\pi$ that seems specific to $\chi_{3}^{(1)}$, the similarity between the three cubic susceptibilities reported in Figs. 1 and 2 puts strong constraints on the underlying physical mechanisms leading to an increase of the peak height with temperature.

\section{ACCOUNTING FOR EXPERIMENTAL RESULTS WITHIN THE FRAMEWORK OF BB}

We now briefly explain why the aforementioned findings are in fact consistent with the theoretical framework put forward by $\mathrm{BB}[11]$. The idea is that provided $f \gtrsim f_{\alpha}$, i.e., for processes faster than the relaxation time, one cannot distinguish between a truly frozen glass and a still flowing liquid. If some amorphous order is present in the glass phase, then nontrivial spatial correlations should be present and lead to anomalously high values of nonlinear susceptibilities. If these spatial correlations extend far enough to be in a scaling regime, one expects the nonlinear susceptibilities to be dominated by the glassy correlations and given by $[11,18]$

$$
X_{2 k+1}^{\text {glass }}(f, T)=\left[N_{\text {corr }}(T)\right]^{k} \mathcal{H}_{k}\left(\frac{f}{f_{\alpha}}\right),
$$

where the scaling functions $\mathcal{H}_{k}$ do not explicitly depend on temperature, but depend on the kind of susceptibility that is considered, i.e., $X_{3}^{(1)}, X_{3}^{(3)}$, or $X_{2,1}^{(1)}$ in the cubic case $k=1$. Since this glassy contribution has been shown to be the most divergent one [11,21], it should dominate over the other contributions to $X_{2 k+1}$ as long as one does not enter the low-frequency regime $f \ll f_{\alpha}$. In the latter regime, relaxation has happened everywhere in the system, destroying amorphous order [30] and the associated anomalous response to the external field and $\mathcal{H}_{k}(0)=0$. In other words, in this very-low-frequency regime, every molecule behaves independently of the others and $X_{2 k+1}$ is dominated by the trivial Langevin response of effectively independent molecules (see [28] for a refined discussion). Due to the definition adopted in Eq. (4), the trivial contribution to $X_{2 k+1}$ should not depend on temperature (or very weakly). Hence, provided $N_{\text {corr }}$ increases upon cooling, there will be a regime where the glassy contribution $X_{2 k+1}^{\text {glass }}$ should exceed the trivial contribution, leading to humped-shape nonlinear susceptibilities, peaking at $f_{\text {peak }} \sim f_{\alpha}$, where the scaling functions $\mathcal{H}_{k}$ reaches its maximum. Focusing on the three salient experimental facts discussed in the preceding section, we find that (i) due to the fact that $\mathcal{H}_{k}$ does not depend explicitly on $T$, the value of $f_{\text {peak }} / f_{\alpha}$ should not depend on temperature, consistently with the experimental behavior; (ii) because of the dominant role played by the glassy response for $f \geqslant f_{\text {peak }}$, the $T$ dependence of $X_{2 k+1}$ will be much stronger above $f_{\text {peak }}$ than in the trivial low-frequency region; and (iii) because nonlinear susceptibilities are expressed in terms of scaling functions, it is natural that the behavior of their moduli and phases are quantitatively related, especially at high frequency where the trivial contribution can be neglected, consistently with Eqs. (5) and (6) (see below for a more quantitative argument in the context of the so-called toy model) [31].

Let us again emphasize that the $\mathrm{BB}$ prediction relies on a scaling argument, where the correlation length $\ell$ of amorphously ordered domains is (much) larger than the molecular size $a$. This naturally explains the similarities of the cubic responses in microscopically very different liquids such as glycerol and propylene carbonate, as well as many other liquids $[13,15]$. Indeed, the microscopic differences are likely to be wiped out for large $\ell$, much like in usual phase transitions.

Throughout this paper, we will not interpret $N_{\text {corr }}$ as a purely dynamical correlation volume, but as a static correlation volume, elicited by a quasistatic nonlinear response [the frequency of the hump is indeed often lower than $f_{\alpha}$ (see Sec. II)]. This interpretation may seem surprising at first sight since theorems relating (in a strict sense) nonlinear responses to high-order correlations functions only exist in the static case and therefore cannot be straightforwardly used to interpret the humped shape of $X_{3}$ (and of $X_{5}$ ) observed experimentally.

This is why each theory of the glass transition must be inspected separately [18] to see whether or not it can account for the anomalous behavior of nonlinear responses observed in frequency and in temperature. The case of the family of $\mathrm{KCM}$ is especially interesting since dynamical correlations, revealed by, e.g., four-point correlation functions, exist even in the absence of a static correlation length. However, in the KCM family, we do not expect any humped shape for nonlinear responses [18]. This is not the case for theories (such as RFOT or frustration theories) where a nontrivial thermodynamic critical point drives the glass transition: In this case the incipient amorphous order allows one to account [18] for the observed features of $X_{3}$ and $X_{5}$. This is why we think that in order for $X_{3}$ to grow some incipient amorphous order is needed and we expect dynamical correlations in strongly supercooled liquids to be driven by static (point-to-set) correlations [32]; this statement will be reinforced by what we find in Sec. IV B. 
Notably, we find that the temperature dependence of $N_{\text {corr }}$ inferred from the height of the humps of the three $X_{3}$ 's are compatible with one another and closely related to the temperature dependence of $T \chi_{T}$, which was proposed in Refs. [16,17] as a simplified estimator of $N_{\text {corr }}$ in supercooled liquids. The convergence of these different estimates, which rely on general model-free theoretical arguments, is a strong hint that the underlying physical phenomenon is indeed the growth of collective effects in glassy systems, a conclusion that we will reinforce by analyzing other approaches.

\section{OTHER PHENOMENOLOGICAL APPROACHES}

A valid criticism of the general $\mathrm{BB}$ prediction is that the analytical expression of the scaling functions $\mathcal{H}_{k}$ is unknown, except for $k=1$ within mode-coupling theory (MCT), where some analytical progress is possible [21]. In particular, only the $T$ dependence of $N_{\text {corr }}$ can be extracted from the experiments using Eq. (7), but not its absolute value. Moreover, since Eq. (7) is only valid in the limit $N_{\text {corr }} \gg 1$, it may happen that other subleading contributions to $X_{3}^{\text {glass }}$ are relevant in the limited range of temperatures available in practice. Some simplified schematic models have therefore been proposed to compute explicitly the different cubic susceptibilities. We show that in each of them $N_{\text {corr }}$ is a key ingredient, either explicit or implicit. For the sake of brevity, we concentrate on physical arguments and postpone further analytical developments to the Appendix; however, we recall some quantitative limitations in the next section. Sections IV A and IV C give definitely phenomenological descriptions, whereas Sec. IV B starts from a solid thermodynamical relation recently pinpointed by Johari, which, when coupled with the well-known Adam-Gibbs relation, provides a physically motivated specification of BB's mechanism.

\section{A. Toy model and pragmatic model}

The toy model has been proposed in Refs. [27,33] as a simple incarnation of BB's mechanism, while the pragmatic model is more recent $[34,35]$. Both models start with the same assumptions: (i) Each amorphously ordered domain containing $N_{\text {corr }}$ molecules has a dipole moment proportional to $\sqrt{N_{\text {corr }}}$, leading to an anomalous contribution to the cubic response $X_{3}^{\text {glass }} \propto N_{\text {corr }}$, and (ii) there is a crossover at low frequencies towards a trivial cubic susceptibility contribution $X_{3}^{\text {triv }}$ that does not depend on $N_{\text {corr }}$. We note in passing that the toy model predicts generally [33] an anomalous contribution $X_{2 k+1}^{\text {glass }} \propto\left[N_{\text {corr }}\right]^{k}$. This naturally accounts for the results of Ref. [18], where it was shown that $X_{5}^{\text {glass }} \propto\left[X_{3}^{\text {glass }}\right]^{2}$ in glycerol and propylene carbonate, consistently with BB's predictions summarized in Eq. (7).

More precisely, in the toy model each amorphously ordered domain is supposed to exist in a simplified energy landscape, namely, an asymmetric double-well potential with a dimensionless asymmetry $\delta$, favoring one well over the other. The most important difference between the toy and the pragmatic model is the description of the low-frequency crossover (see Refs. [33,35] for more details).

On top of $N_{\text {corr }}$ and $\delta$, the toy model uses a third adjustable parameter, namely, the frequency $f^{*}$ below which the trivial contribution becomes dominant. In Ref. [33], both the modulus and the phase of $X_{3}^{(1)}(\omega, T)$ and of $X_{3}^{(3)}(\omega, T)$ in glycerol were well fitted by using $f^{*} \simeq f_{\alpha} / 7, \delta=0.6$, and, for $T=204 \mathrm{~K}$, $N_{\text {corr }}=5$ for $X_{3}^{(3)}$ and $N_{\text {corr }}=15$ for $X_{3}^{(1)}$. In Ref. [27], the behavior of $X_{2,1}^{(1)}(\omega, T)$ in glycerol was further fitted with the same values of $\delta$ and of $f^{*}$ but with $N_{\text {corr }}=10$ (at a slightly different temperature $T=202 \mathrm{~K}$ ). Of course, the fact that a different value of $N_{\text {corr }}$ must be used for the three cubic susceptibilities reveals that the toy model is oversimplified, as expected. However, keeping in mind that the precise value of $N_{\text {corr }}$ does not change the behavior of the phases, we note that the fit of the three experimental phases is achieved [27,33] by using the very same values of $f^{*} / f_{\alpha}$ and of $\delta$. This means that Eqs. (5) and (6) are well accounted for by the toy model by choosing two free parameters. This is a quantitative illustration of how BB's general framework does indeed lead to strong relations between the various nonlinear susceptibilities, such as those contained in Eqs. (5) and (6).

Let us mention briefly the asymmetric double-well potential model [36], which is also about species existing in a double well of asymmetry energy $\Delta$, excepted that two key assumptions of the toy and pragmatic models are not made: The value of $N_{\text {corr }}$ is not introduced and the crossover to trivial cubic response is not enforced at low frequencies. As a result, the hump for $\left|X_{3}^{(3)}\right|$ is predicted $[36,37]$ only when the reduced asymmetry $\delta=\tanh \left(\Delta / 2 k_{B} T\right)$ is close to a very specific value, namely, $\delta_{c}=\sqrt{1 / 3}$, where $X_{3}$ vanishes at zero frequency due to the compensation of its several terms. However, at the fifth order [37] this compensation happens for two values of $\delta$ very different from $\delta_{c}$ : as a result, the model cannot predict a hump happening both for the third and for the fifth order in the same parametric regime, contrarily to the experimental results of Ref. [18]. This very recent calculation of fifth-order susceptibility [37] reinforces the point of view of the toy and pragmatic models, which do predict a hump occurring at the same frequency and temperature due to their two key assumptions ( $N_{\text {corr }}$ and crossover to trivial nonlinear responses at low frequencies). This can be understood qualitatively: Because the toy model predicts [33] an anomalous contribution $X_{2 k+1}^{\text {glass }} \sim\left[N_{\text {corr }}\right]^{k}$, provided that $N_{\text {corr }}$ is large enough, the magnitude of this contribution is much larger than that of the small trivial contribution $X_{2 k+1}^{\text {triv }} \sim 1$, and the left side of the peak of $\left|X_{2 k+1}\right|$ arises just because the toy model enforces a crossover from the large anomalous response to the small trivial response at low frequencies $f \ll f_{\alpha}$. As for the right side of the peak, it comes from the fact that $\left|X_{2 k+1}\right| \rightarrow 0$ when $f \gg f_{\alpha}$ for the simple reason that the supercooled liquid does not respond to the field at very large frequencies.

\section{B. Entropic effects}

We recall the argument given by Johari in [38,39]. Suppose a static electric field $E_{\mathrm{st}}$ is applied to a dielectric material at temperature $T$. By using the general relations of thermodynamics, one finds that a variation of entropy $[\delta S]_{E_{\text {st }}}$ follows, which for small $E_{\mathrm{st}}$ is given by

$$
[\delta S]_{E_{\mathrm{st}}} \approx \frac{1}{2} \epsilon_{0} \frac{\partial \Delta \chi_{1}}{\partial T} E_{\mathrm{st}}^{2} a^{3},
$$


where $a^{3}$ is the molecular volume. Equation (8) holds generically for any material. However, in the specific case of supercooled liquids close enough to their glass transition temperature $T_{g}$, a special relation exists between the molecular relaxation time $\tau_{\alpha}$ and the configurational contribution to the entropy $S_{c}$. This relation, first anticipated by Adam and Gibbs [2], can be written as

$$
\ln \frac{\tau_{\alpha}(T)}{\tau_{0}}=\frac{\Delta_{0}}{T S_{c}(T)},
$$

where $\tau_{0}$ is a microscopic time and $\Delta_{0}$ is an effective energy barrier for a molecule. The temperature dependence of $T S_{c}(T)$ quite well captures the temperature variation of $\ln \left(\tau_{\alpha}\right)$, at least for a large class of supercooled liquids [40].

We now follow Johari $[38,39]$ and we assume that $[\delta S]_{E_{\text {st }}}$ is dominated by the dependence of $S_{c}$ on field; see the Appendix, Sec. 2 for further discussion of this important physical assumption. Combining Eqs. (8) and (9), we find that a static field $E_{\text {st }}$ produces a shift of $\ln \left(\tau_{\alpha} / \tau_{0}\right)$ given by

$$
\left[\delta \ln \tau_{\alpha}\right]_{E_{\mathrm{st}}}=-\frac{\Delta_{0}}{T S_{c}^{2}}[\delta S]_{E_{\mathrm{st}}} .
$$

We show in the Appendix, Sec. 2 that this entropic effect gives a contribution to $X_{2,1}^{(1)}$, which we call $J_{2,1}^{(1)}$ after Johari. Introducing $x=\omega \tau_{\alpha}$, we obtain

$$
J_{2,1}^{(1)}=-\frac{k_{B} \Delta_{0}}{6 S_{c}^{2}}\left[\frac{\partial \ln \left(\Delta \chi_{1}\right)}{\partial T}\right]\left[\frac{\partial \frac{\chi_{\operatorname{lin}}}{\Delta \chi_{1}}}{\partial \ln x}\right] \propto \frac{1}{S_{c}^{2}},
$$

where $\chi_{\operatorname{lin}}$ is the complex linear susceptibility.

Equation (11) deserves two comments. First, $\left|J_{2,1}^{(1)}\right|$ has a humped shaped in frequency with a maximum in the region of $\omega \tau_{\alpha} \simeq 1$, because of the frequency dependence of the factor proportional to $\partial \chi_{\operatorname{lin}} / \partial \ln x$ in Eq. (11). Second, the temperature variation of $J_{2,1}^{(1)}$ is overwhelmingly dominated by that of $S_{c}^{-1}$ because $S_{c} \propto T-T_{K}$, with $T_{K}$ the Kauzman temperature.

In fact, following Adam and Gibbs' original formulation [2], the dynamics of a supercooled liquid comes from cooperatively rearranging regions (CRRs). Assuming that these regions are compact (see [7,41]; see also [18] for a recent discussion of this point), the spatial extension $\ell$ of the CRRs is related to the number $N_{\text {corr }}$ of molecules as $N_{\text {corr }}=(\ell / a)^{d}$, where $d$ is the dimensionality of space. Within the Adam-Gibbs picture, $S_{c} \propto 1 / N_{\text {corr }}$, leading to

$$
J_{2,1}^{(1)} \propto N_{\text {corr }}^{q},
$$

with $q=2$, i.e., a stronger divergence than predicted by BB, but a similar qualitative relation between nonlinear effects and glassy correlations. Taking into account more general relationships between $S_{c}$ and $N_{\text {corr }}$, we find that the possible values of $q$ are bounded between $2 / 3$ and 2 (see the Appendix, Sec. 3 a).

However, the Adam-Gibbs picture has been reformulated more convincingly within the RFOT theory of glasses (see $[7,42]$ ). This leads to more constrained results (see the Appendix, Sec. 3 b)

$$
J_{2,1}^{(1)} \propto N_{\text {corr }}^{q}, \quad q=1+\frac{\Psi-\theta}{d},
$$

where $\Psi$ is the barrier exponent and $\theta$ the surface tension exponent (see the Appendix, Sec. 3 b). We note in passing that formally, the Adam-Gibbs picture corresponds to $\theta=0$ and $\Psi=d$. Returning to the RFOT theory, the exponents $\theta$ and $\Psi$ should obey, in general, the following bounds:

$$
\frac{d}{2} \leqslant \theta \leqslant d-1 .
$$

The upper bound is natural for a surface-tension exponent, whereas the lower bound is obtained if one takes into account the existence of self-induced disorder: If $\theta<d / 2$ amorphous order would be destroyed by the disorder, which is what one expects below the lower critical dimension. Concerning $\Psi$, arguments based on the free-energy landscape give $\Psi \geqslant \theta$ [42]. However, it is possible that these do not hold for the dynamical rearrangements responsible for relaxation; indeed, numerical results seem to favor $\psi<\theta$ [43]. From these bounds, one concludes [44] that, for $d=3, q$ lies in the range [1/3,3/2], where $q=1$ corresponds to the recommended RFOT values, $\Psi=\theta=d / 2$. Note that $q=1$ precisely corresponds to BB's prediction, in which case entropic effects are a physically motivated picture of BB's mechanism. For the lowest values of $q \simeq 1 / 3$, the Johari contribution is actually expected to be really subleading with respect to BB's contribution. Indeed, in BB's framework, the only way for $X_{3}$ to grow slower than $N_{\text {corr }}$ is that glassy domains are noncompact [18], a possibility that is difficult to accommodate both with the RFOT theory and with the experimental results of Ref. [18].

To summarize this section, the two key assumptions for computing Johari's entropy effect are that the field-induced entropy variation mainly goes into the configurational part of the entropy and that its effect can be evaluated by using the Adam-Gibbs relation. We have found that the entropy contribution to $X_{2 ; 1}^{(1)}$, called $J_{2 ; 1}^{(1)}$, is similar to BB's general prediction both because of its humped shape in frequency [see Eq. (11)] and because it is directly related to $N_{\text {corr }}$ [see Eqs. (12) and (13)]. Additionally, because $S_{c}$ is a static quantity, Eqs. (12) and (13) support our interpretation that $X_{3}$ is related to static amorphous correlations, as stated at the end of Sec. III.

Let us add two remarks. One is about the extension of the above calculation to $X_{3}^{(1)}$ and to $X_{3}^{(3)}$. Such an extension is a key ingredient of the phenomenological model elaborated in Refs. $[22,23]$ and gives the main term allowing one to fit the modulus of $X_{3}^{(3)}$ for glycerol [23]. This extension came after several works [45-48] where the entropic effects were found to be consistent with the measured $X_{2 ; 1}^{(1)}$ in various systems. Note that to perform this extension one needs to introduce a time-dependent configurational entropy, which is nevertheless acceptable in the region $\omega \tau_{\alpha} \lesssim 1$, where the model is used. The second remark is that there must be other contributions to $X_{2,1}^{(1)}$ coming from, e.g., the field dependence of the energy barrier $\Delta_{0}$ or of the surface energy cost $\Upsilon$ in the RFOT. Following the calculations in Appendix, Sec. $3 \mathrm{~b}$, this leads to a contribution that behaves as $N_{\text {corr }}^{\Psi / d}$, which is subdominant compared to $J_{2,1}^{(1)}$ as given by Eq. (13). This illustrates that between BB's leading contribution to $X_{2 ; 1}^{(1)}$ and the mere trivial contribution, there is room for intermediate terms scaling more slowly than $N_{\text {corr }}$. 


\section{Box model}

The box model is historically the first model of nonlinear response in supercooled liquids designed to account for the nonresonant hole burning experiments [49]. It assumes [4953] that some heterogeneous heating happens within each of the amorphously ordered domain and that the thermal time during which the dissipated heat is kept within each domain is as long as the dielectric relaxation time of the domain, i.e., as long as seconds when $T$ is close to $T_{g}$. According to Ref. [35], this seems to contradict physical intuition, since the size of amorphously ordered domains is only a few nanometers [54]. The box model has been shown to give good fits of the imaginary part of $X_{3}^{(1)}$ for $f>f_{\alpha}$ in many glass-forming liquids (see, e.g., [50-53]). It was shown also [55] that the box model is not able to fit quantitatively the measured $X_{3}^{(3)}$ (even though some qualitative features are accounted for) and that the box model only provides a vanishing contribution to $X_{2,1}^{(1)}[27]$.

In recent works [22,23], the three experimental cubic susceptibilities have been argued to result from a superposition of an entropic contribution and of a contribution coming from the box model, plus a trivial contribution playing a minor role around the peaks of the cubic susceptibilities. More precisely, the hump of $\left|X_{2,1}^{(1]}\right|$ and of $\left|X_{3}^{(3)}\right|$ would be mainly due to the entropy effect, contrarily to the hump of $\left|X_{3}^{(1)}\right|$, which would be due to the box model contribution. This means that very different physical mechanisms would conspire to give contributions of the same order of magnitude, with phases that have no reason to match as they do empirically [see Eqs. (5) and (6)]: Why should $X_{3}^{(1)}$ and $X_{3}^{(3)}$ have the same phase at high frequencies if their physical origin is different?

We see no reason for such a similarity if the growth of $X_{3}^{(1)}$ and $X_{3}^{(3)}$ is due to independent mechanisms. Having just related entropic effects to the increase of $N_{\text {corr }}$, everything becomes instead very natural if the box model is recast in a framework where $X_{3}^{(1)}$ is related to the glassy correlation volume. As a first step in this direction, we show in the Appendix, Sec. 4 that the box model prediction for $X_{3}^{(1)}$ at high frequencies is proportional to the above toy model prediction, provided $N_{\text {corr }}$ and $T \chi_{T}$ are proportional, which is a reasonable assumption, as explained at the end of Sec. III and in Refs. [16,17,24]. Overall, the only reasonable way to account for the similarity of all three cubic susceptibilities, demonstrated experimentally in Sec. II, is to invoke a common physical mechanism. As all the other existing approaches previously reviewed in this paper relate cubic responses to the growth of the glassy correlation volume, reformulating the box model along the same line is, in our view, a necessity.

\section{CONCLUSION}

We have compared three different cubic susceptibilities, in two different liquids, and found that they all behave very similarly in frequency and in temperature, both for their moduli and for their phases. This suggests a unique underlying physical mechanism, which we argue is the growth of a glassy correlation length, measuring the size of the domains where amorphous order sets in. The theoretical framework proposed by two of us [11] (BB) provides a consistent description for all cubic (and higher-order [18]) susceptibilities $X_{3}$. We have reviewed various phenomenological models that attempt to give a quantitative description of $X_{3}$. Although some of them are at first sight not compatible with the previous scenario and lead to puzzling physical predictions compared to our experiments, we explained why they are not in contradiction with BB's predictions. Excepted for the box model where the task is not fully achieved, all the models can be actually recast in such a way that the number of correlated molecules $N_{\text {corr }}$ appears (implicitly or explicitly) as a key ingredient.

Having unified various approaches of nonlinear responses close to $T_{g}$, our work opens at least two routes of research. First, it would be very interesting to access $\chi_{3}$ (and $\chi_{5}$ ) in molecular liquids at higher temperatures, closer to the MCT transition, and/or for frequencies close to the fast $\beta$ process where more complex fractal structures with $d_{f}<d$ may be anticipated [6,56]. Note that even though $X_{3}^{(1)}$ or $X_{3}^{(3)}$ is plagued by heating issues when $f_{\alpha}$ is large, this is not the case for $X_{2,1}^{(1)}$ because a dc field yields negligible dissipation. As we have shown that the three cubic susceptibilities are driven by the same physics, it would be wise to choose $X_{2,1}^{(1)}$ to investigate the behavior of molecular liquids at high temperatures. Second, we could revisit the vast field of polymers by monitoring their nonlinear responses, which should shed light on the temperature evolution of the correlations in these systems. Therefore, we think that there is much room to deepen our understanding of the glass transition by carrying out experiments about nonlinear susceptibilities.

\section{ACKNOWLEDGMENTS}

We thank C. Alba-Simionesco, U. Buchenau, A. Coniglio, P.-M. Déjardin, R. Richert, G. Tarjus, and M. Tarzia for interesting discussions. This work in Saclay was supported in part by ERC Grant NPRGLASS, by the Simons Foundation through Grant No. 454935 (G.B.), by the Labex RTRA grant Aricover, and by the Institut des Systèmes Complexes. This work in Augsburg was supported by the Deutsche Forschungsgemeinschaft via Research Unit FOR1394.

\section{APPENDIX}

\section{The spikelike minimum in $\left|X_{3}^{(1)}\right|$ for propylene carbonate}

The spikelike minimum as indicated by the red line in Fig. 2(a) seems somewhat speculative. However, when plotting the same data set as real and imaginary parts (see Fig. 3), it becomes obvious that $\left|X_{3}^{(1)}\right|$ becomes zero at a certain frequency, thus generating a negative spike in the logarithmic plot of Fig. 2(a): When the trivial response starts to dominate at low frequencies, the real part of $X_{3}^{(1)}$ must become negative because dielectric saturation causes a decrease of the dielectric constant $\chi_{\text {lin }}^{\prime}$ at high fields [i.e., negative $\operatorname{Re}\left(X_{3}^{(1)}\right)$ ] instead of the increase seen at higher frequencies [positive $\operatorname{Re}\left(X_{3}^{(1)}\right)$ ]. This causes $\operatorname{Re}\left(X_{3}^{(1)}\right)$ to cross the zero line. The imaginary part of $X_{3}^{(1)}$ also is close to zero in this region and thus the modulus $\left|X_{3}^{(1)}\right|$ also becomes extremely small at this crossover frequency. 


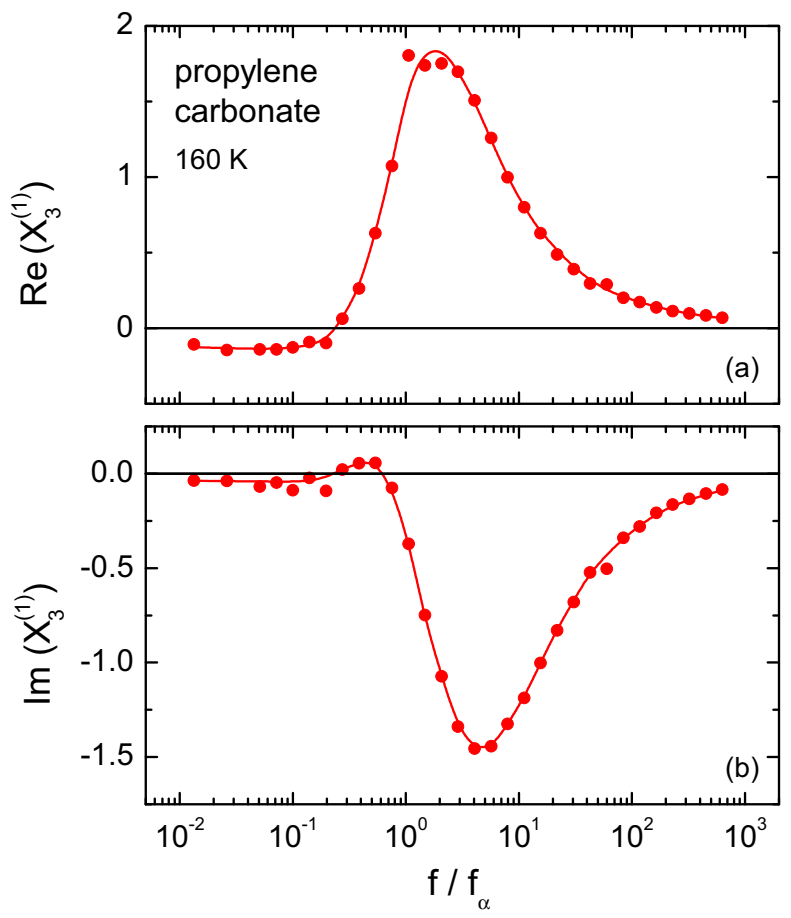

FIG. 3. Real and imaginary parts of the $X_{3}^{(1)}$ data of propylene carbonate shown in Fig. 2. Lines are guides for the eyes.

\section{Entropic effects}

The argument of Johari is to decompose the total entropy $S_{\text {tot }}$ of a supercooled liquid in its vibrational part $S_{\text {vib }}$ and its configurational part $S_{c}$. Then, because of the smallness of electrostriction effects in general $[38,39]$, Johari deduces that the field-induced variation of $S_{\text {vib }}$ is much smaller than that of $S_{c}$. We note that this argument can be reinforced by an alternative reasoning: $S_{\text {tot }}$ can also be decomposed as the entropy of the crystal $S_{\text {cryst }}$ plus an excess entropy $S_{\text {exc }}$, where $S_{\text {exc }}$ contains the configurational entropy $S_{c}=f_{S} S_{\text {exc }}$; the factor $f_{S}<1$ does not depend on the temperature [57] and will be disregarded hereafter. As for some archetypical glass formers, such as glycerol and propylene carbonate studied in the Sec. II B, the static value of the dielectric linear susceptibility $\Delta \chi_{1}$ is much larger in the supercooled liquid than in the crystal, it is very likely that $\partial \Delta \chi_{1} / \partial T$ is also much larger in the supercooled liquid than in the crystal. With the help of Eq. (8), this means that the field-induced variation of $S_{\text {cryst }}$ is much smaller than that of $S_{\text {exc }}$ and thus also of $S_{c}$. Of course the validity of this alternative argument is restricted to the subclass of glass formers where $\Delta \chi_{1} \gg 1$ in the supercooled liquid state.

We now focus specifically on the case where an ac field $E_{\mathrm{ac}}$, of angular frequency $\omega$, is applied on top of the static field $E_{\mathrm{st}}$. Consistently with the assumption of a linear dielectric response allowing us to derive Eq. (8), we express the polarization $P$ when $E_{\mathrm{st}}=0$ as $P\left(E_{\mathrm{ac}}, 0, T\right)=\epsilon_{0} \chi_{\mathrm{lin}} E_{\mathrm{ac}}$, where $\chi_{\mathrm{lin}}$ is a complex quantity that, once scaled by $\Delta \chi_{1}$, depends mainly on $x=\omega \tau_{\alpha}$. Considering Eq. (10), we obtain

$$
P\left(E_{\mathrm{ac}}, E_{\mathrm{st}}, T\right)=P\left(E_{\mathrm{ac}}, 0, T\right)+\frac{\partial P}{\partial \ln \tau_{\alpha}}\left[\delta \ln \tau_{\alpha}\right]_{E_{\mathrm{st}}}+\cdots,
$$

where higher-order terms in $\delta \ln \tau_{\alpha}$ have been neglected. Because $P\left(E_{\mathrm{ac}}, 0, T\right) \propto E_{\mathrm{ac}}$ and $\delta \ln \tau_{\alpha} \propto E_{\mathrm{st}}^{2}$, defining $\delta P_{E_{\mathrm{st}}}=$ $P\left(E_{\mathrm{ac}}, E_{\mathrm{st}}, T\right)-P\left(E_{\mathrm{ac}}, 0, T\right)$, one finds $\delta P_{E_{\mathrm{st}}} \propto E_{\mathrm{st}}^{2} E_{\mathrm{ac}}$. This shows that $\delta P_{E_{\mathrm{st}}}$ is cubic in the electric field.

Inserting Eq. (8) into Eq. (10) and using Eq. (A1), we find that the entropy variation induced by the static electric field yields a term proportional to $E_{\mathrm{st}}^{2} E_{\mathrm{ac}}$, i.e., it yields a contribution to $X_{2,1}^{(1)}$ because of the definition given in Eq. (2). We obtain for this entropic contribution to $\chi_{2 ; 1}^{(1)}$,

$$
\chi_{2,1}^{(1), \delta S}=\left[-\frac{1}{6}\right] \epsilon_{0}\left[\frac{\partial \Delta \chi_{1}}{\partial T}\right] a^{3} \frac{\Delta_{0}}{S_{c}^{2} T}\left[\frac{\partial \chi_{\operatorname{lin}}}{\partial \ln x}\right],
$$

where we recall our notation $x \equiv \omega \tau_{\alpha}$.

By using Eq. (A2) we find the entropy contribution $J_{2,1}^{(1)}$ to the dimensionless cubic susceptibility $X_{2,1}^{(1)}$,

$$
J_{2,1}^{(1)}=-\frac{k_{B} \Delta_{0}}{6 S_{c}^{2}}\left[\frac{\partial \ln \left(\Delta \chi_{1}\right)}{\partial T}\right]\left[\frac{\partial \frac{\chi_{\operatorname{lin}}}{\Delta \chi_{1}}}{\partial \ln x}\right] \propto \frac{1}{S_{c}^{2}},
$$

where, as explained above, $\chi_{\text {lin }}$ is a complex quantity. As explained in the main text, Eq. (A3) implies that $\left|J_{2 ; 1}^{(1)}\right|$ is peaked for a frequency close to $f_{\alpha}$. We note that in an ideal gas of dipoles $\left|X_{2 ; 1}^{(1)}\right|$ has no peak at any frequency (see the works cited in [28]). This comes from the fact that in an ideal gas of dipoles the relaxation time is insensitive to the static field, i.e., the last term of Eq. (A1) has to be neglected when computing $X_{2 ; 1}^{(1)}$. Assuming a nonzero value of $\left[\delta \ln \tau_{\alpha}\right]_{E_{\mathrm{st}}}$ is thus a highly nontrivial assumption that calls for an explanation. We have argued, following Johari, that $\left[\delta \ln \tau_{\alpha}\right]_{E_{\text {st }}}$ comes from entropic effects. In the case where this could be disputed, e.g., when $\Delta \chi_{1} \simeq 1 \mathrm{and} /$ or when the factor $f_{S}$ turns out to be very far from 1 , we show briefly that a nonzero value of $\left[\delta \ln \tau_{\alpha}\right]_{E_{\mathrm{st}}}$ is related to the glassy correlation volume. Indeed, one has

$$
\left[\delta \ln \tau_{\alpha}\right]_{E_{\mathrm{st}}}=\left[\frac{\partial \ln \tau_{\alpha}}{\partial E_{\mathrm{st}}^{2}}\right]_{T} E_{\mathrm{st}}^{2}=-\left[\frac{\partial \ln \tau_{\alpha}}{\partial T}\right]_{E_{\mathrm{st}}=0} \mathcal{M} E_{\mathrm{st}}^{2},
$$

where $\mathcal{M}=\partial T_{g} / \partial E_{\mathrm{st}}^{2}$ expresses the shift of the glass transition temperature $T_{g}$ induced by $E_{\mathrm{st}}$, i.e., the fact that the dielectric spectrum is uniformly shifted in frequency by the static field; the minus sign in the last equality of Eq. (A4) comes from the mapping between $P\left(T, E_{\mathrm{st}}\right)$ and $P\left(T-\mathcal{M} E_{\mathrm{st}}^{2}, 0\right)$ (see [27]). As a result,

$$
\left[\frac{\partial \ln \tau_{\alpha}}{\partial E_{\mathrm{st}}^{2}}\right]_{T}=-\mathcal{M}\left[\frac{\partial \ln \tau_{\alpha}}{\partial T}\right]_{E_{\mathrm{st}}=0}=\frac{\mathcal{M}}{T}\left|T \chi_{T}\right|,
$$

which establishes that a nonzero value of $\left[\delta \ln \tau_{\alpha}\right]_{E_{s t}}$ must be related to $T \chi_{T}$, i.e., to the glassy correlation volume, as advocated in Refs. [16,17]. Having briefly evoked the case where the origin of the nonzero value of $\left[\delta \ln \tau_{\alpha}\right]_{E_{\mathrm{st}}}$ is questionable, we now return to the case where this origin is the entropy effect pinpointed by Johari.

\section{Relation between configurational entropy and length scales}

\section{a. Adam-Gibbs case}

When lowering $T$, a supercooled liquid becomes increasingly viscous and its dynamics comes from "cooperatively 
rearranging regions," to quote the original expression of Adam and Gibbs [2]. Expanding on [18], as well as on several theoretical approaches $[7,41]$, we will assume that these regions are compact, i.e., their spatial extension $\ell$ is related to their number $N_{\text {corr }}$ of molecules by $N_{\text {corr }}=(\ell / a)^{d}$, where $d$ is the dimensionality of space.

Returning to the original argument of Adam and Gibbs [2] readily gives a lower bound for $N_{\text {corr }}$. Indeed, owing to its extensive character, the configurational entropy of a domain of size $\ell$ is $S_{c}(\ell / a)^{d}$. For this domain to be able to relax, at least two states must be available and thus the aforementioned configurational entropy cannot be smaller than $k_{B} \ln 2$. As a result,

$$
\left(\frac{\ell}{a}\right)^{d} \geqslant \frac{k_{B} \ln 2}{S_{c}}, \quad \frac{k_{B} \ln 2}{S_{c}} \leqslant N_{\text {corr }} .
$$

In addition, by using Refs. [42,58,59], one can find an upper bound for $N_{\text {corr }}$. For a given domain of size $\ell$ where $N_{\text {corr }}$ molecules relax cooperatively, the argument comes from the comparison of the number of accessible states $\mu S_{c}(\ell / a)^{d}$ with the number of different boundary conditions $\lambda(\ell / a)^{d-1}$ (here $\mu$ and $\lambda$ are constants). The latter must be larger than the former; otherwise there are not enough boundary conditions to select each of the accessible states. This would mean that when freezing all the molecules of the system except those inside the domain with size $\ell$, one cannot define a preferred state, since many states are possible. This contradicts the assumption that within the considered domain all the molecules are in a well-defined state. To avoid this contradiction, one needs to write

$$
\lambda\left(\frac{\ell}{a}\right)^{d-1} \geqslant \mu S_{c}\left(\frac{\ell}{a}\right)^{d}, \quad N_{\text {corr }} \leqslant\left[\frac{\lambda}{\mu S_{c}}\right]^{d} .
$$

By using the two boundaries obtained in Eqs. (A6) and (A7), we get, with $d=3$,

$$
\frac{1}{S_{c}^{2}}=\Gamma\left[N_{\text {corr }}\right]^{q} \text { with } \frac{2}{3} \leqslant q \leqslant 2,
$$

where both the proportionality constant $\Gamma$ and the exponent $q$ should not depend on temperature owing to the fact that $S_{c}$ and $N_{\text {corr }}$ are the only temperature-dependent quantities in the aforementioned inequalities. We now combine Eqs. (A8) and (A3) to obtain

$$
J_{2,1}^{(1)} \propto\left[N_{\text {corr }}\right]^{q} \quad \text { with } \frac{2}{3} \leqslant q \leqslant 2 .
$$

We emphasize that as the exponent $q$ cannot be zero, Eq. (A9) establishes that the aforementioned entropic contribution to the cubic susceptibility is connected to $N_{\text {corr }}$.

\section{b. Specific case of RFOT}

The highest possible value $q=2$ in Eqs. (A8) and (A9) corresponds to the Adam-Gibbs argument $S_{c} \propto 1 / N_{\text {corr }}$. In the original Adam-Gibbs argument $[2,42,60]$, this comes from the assumption that the barrier height $\Delta$ governing relaxation is proportional to $N_{\text {corr }}=(\ell / a)^{d}$. This of course overestimates $\Delta$ since it supposes that any relaxation involves a finite fraction of the total number of molecules in the domain. This is unlikely when $\ell$ is large since, in general, the energy cost to relax a domain of size $\ell$ cannot scale more rapidly than $(\ell / a)^{(d-1)}$ (see [61]).

It does not seem easy to combine this idea that the energy cost scales proportionally to $(\ell / a)^{\theta}$, where $\theta$ must be lower than or equal to $d-1$ with the result that $\ln \left(\tau_{\alpha} / \tau_{0}\right) \propto 1 / T S_{c}$, which, as already mentioned, is obeyed in a vast series of glassforming liquids [40]. The random first-order transition theory [7], proposed 30 years after the Adam-Gibbs seminal paper, achieves this task, as we briefly recall now. According to the RFOT theory, if a domain of size $\ell$ relaxes, the associated freeenergy cost is given by $\delta F=\Upsilon(\ell / a)^{\theta}-T S_{c}(\ell / a)^{d}$, where the first term corresponds to the surface energy cost and the second term is the free-energy gain of entropic origin. Relaxation can happen only provided that states are available, i.e., for large enough $\ell$. One shows [42] that this happens when $\ell \geqslant \ell^{\star}$, where $\ell^{\star}$ is the maximum of $\delta F(\ell)$. As the relaxation time $\tau$ is given by $\ln \left(\tau / \tau_{0}\right)=(\ell / a)^{\Psi} \Delta_{0} / k_{B} T$, the typical domain size is $\ell^{\star}$ because it is the one that minimizes $\tau$. One finds

$$
\begin{gathered}
\left(\frac{\ell^{\star}}{a}\right)^{d}=\left[\frac{\theta \Upsilon}{d T S_{c}}\right]^{d /(d-\theta)}, \\
\ln \frac{\tau_{\alpha}}{\tau_{0}}=\frac{\Delta_{0}}{k_{B} T}\left[\frac{\theta \Upsilon}{d T S_{c}}\right]^{\Psi /(d-\theta)} .
\end{gathered}
$$

Using this relation and that

$$
N_{\text {corr }}=\left(\frac{\ell^{\star}}{a}\right)^{d},
$$

one finds Eq. (13) for the entropic contribution.

Within the RFOT theory it has been argued that $\Psi=$ $\theta=d / 2$ and that $\Upsilon=\kappa T$, with $\kappa$ a quasiuniversal constant [7]. This leads to the Adam-Gibbs relation, i.e., $\ln \left(\tau_{\alpha} / \tau_{0}\right) \propto$ $1 / T S_{c}$, and to a $\chi_{2,1}^{(1)}$ that is directly proportional to $N_{\text {corr }}$.

\section{Box model predictions for $X_{3}^{(1)}$}

In the box model, the supercooled liquid is assumed to be made of independent dynamical heterogeneities (DHs). Each DH has its own dielectric relaxation time $\tau_{\mathrm{DH}}$ and the probability distribution $\mathcal{G}$ of the $\tau$ 's is chosen so as to recover the linear polarization of the material. The dynamics of the polarization of a given DH is assumed to be given by a Debye equation, i.e., its time-dependent linear polarization $P_{\text {lin,DH }}(t)$ is given by

$$
P_{\text {lin }, \mathrm{DH}}(t)=\frac{\epsilon_{0} \Delta \chi_{1} E_{\mathrm{ac}}}{\sqrt{1+y^{2}}} \cos [\omega t-\arctan (y)],
$$

where we have set $y=\omega \tau_{\mathrm{DH}}$.

The key assumption of the box model is about the dissipated heat, which is supposed to remain confined within each $\mathrm{DH}$ during a thermal time equal to the dielectric time $\tau_{\mathrm{DH}}$. Because the dissipated power $\mathcal{P}$ is quadratic in the field $E_{\text {ac }}$, it contains a static term $\mathcal{P}_{0}$ and a term $\mathcal{P}_{2}$ oscillating at $2 \omega$. The resulting heating of the considered DH contains the two corresponding terms $\delta T_{0, \mathrm{DH}}$ and $\delta T_{2, \mathrm{DH}}$.

Because the box model has been shown to be efficient only for $X_{3}^{(1)}\left(f \gg f_{\alpha}\right)$ we will focus on $X_{3}^{(1)}$ and use the fact that $f \gg f_{\alpha}$ amounts to $y \gg 1$ for most of the DHs; this comes from the shape of $\mathcal{G}(\tau)$, which has a sharp peak around 
$\tau_{\alpha}=1 / 2 \pi f_{\alpha}$. As shown in Eqs. (4) and (5) of Ref. [55], as soon as $y \gg 1$ one has $\delta T_{2, \mathrm{DH}} \ll \delta T_{0, \mathrm{DH}}$ and $X_{3}^{(1)}$ can be computed just by using the values of $\delta T_{0, \mathrm{DH}}$ in the various DHs. For any given DH where $y \gg 1$ one gets [55]

$$
\begin{gathered}
P_{3, \mathrm{DH}}^{(1)}(t) \simeq \frac{\partial P_{\mathrm{lin}, \mathrm{DH}}(t)}{\partial T} \delta T_{0}, \\
\delta T_{0, \mathrm{DH}}=\frac{\epsilon_{0} \Delta \chi_{1} E_{\mathrm{ac}}^{2}}{2 c_{\mathrm{DH}}} \frac{y^{2}}{1+y^{2}},
\end{gathered}
$$

where $c_{\mathrm{DH}}$ is the part of the (volumetric) specific heat involved in the DHs and $P_{3, \mathrm{DH}}^{(1)}$ is, for a given $\mathrm{DH}$, the term corresponding to the first one on the right-hand side of Eq. (2). In complete analogy with Eqs. (2)-(4), we give here the definition of the dimensionless cubic susceptibility $\left|X_{3, \mathrm{DH}}^{(1)}\right| e^{-i \delta_{3, \mathrm{DH}}^{(1)}}$ for a given $\mathrm{DH}$, namely,

$$
P_{3, \mathrm{DH}}^{(1)}(t)=\frac{3}{4} \epsilon_{0} \frac{\epsilon_{0} \Delta \chi_{1}^{2} a^{3}}{k_{B} T} E_{\mathrm{ac}}^{3}\left|X_{3, \mathrm{DH}}^{(1)}\right| \cos \left(\omega t-\delta_{3, \mathrm{DH}}^{(1)}\right) .
$$

We now just have to combine Eqs. (A12)-(A15) to obtain

$$
\begin{aligned}
\left|X_{3, \mathrm{DH}}^{(1)}\right| e^{-i \delta_{3, \mathrm{DH}}^{(1)}} \simeq & \frac{4}{3} \frac{k_{B}}{c_{\mathrm{DH}} a^{3}}\left|T \chi_{T}\right| \frac{y^{3} \sqrt{4 y^{2}+\left(y^{2}-1\right)^{2}}}{\left(1+y^{2}\right)^{3}} \\
& \times e^{-i \arctan \left[\left(y^{2}-1\right) / 2 y\right]},
\end{aligned}
$$

where we have set $\left|T \chi_{T}\right|=-\partial \ln \left(\tau_{\mathrm{DH}}\right) / \partial \ln (T)$ because we assume that the time-temperature superposition property holds, i.e., that all the $\tau$ evolve in temperature as the typical relaxation time $\tau_{\alpha}$. Apart from that, in Eq. (A16) the approximate inequality comes only from the fact that we have neglected the subleading term in $y$ coming from $\delta T_{2, \mathrm{DH}}$ in Eq. (A13).

We now fully simplify Eq. (A16) by taking the limit $y \gg 1$,

$$
\lim _{y \gg 1}\left|X_{3, \mathrm{DH}}^{(1, \mathrm{BM})}\right| e^{-i \delta_{3, \mathrm{DH}}^{(1, \mathrm{BM})}}=\frac{4}{3} \frac{k_{B}}{c_{\mathrm{DH}} a^{3}}\left|T \chi_{T}\right| \frac{e^{-i \pi / 2}}{y},
$$

where the superscript BM stands for box model to make the distinction from the toy model that we are using hereafter. The toy model, introduced in Ref. [33], starts with the same assumption as the box model regarding the decomposition of a supercooled liquids into independent DHs of distribution $\mathcal{G}(\tau)$. The dynamical equation for the polarization in the toy model is a simple Debye equation for the linear response, but when considering higher-order responses, new nonlinear terms arise in the equation. As explained in the main text, these nonlinear terms do not come from heating at the scale of each DH but from the key assumption of the toy model, i.e., from the assumption that each amorphously ordered $\mathrm{DH}$ has a dipole moment proportional to $\sqrt{N_{\text {corr }}}$. This yields generically $X_{2 k+1} \propto N_{\text {corr }}^{k}$. By using Eq. (A29) of Ref. [33], one has

$$
\begin{aligned}
\left|X_{3, \mathrm{DH}}^{(1, \mathrm{TM})}\right| e^{-i \delta_{3, \mathrm{DH}}^{(1, \mathrm{TM})}=} & {\left[\frac{3}{5}\right] \frac{N_{\mathrm{corr}}}{1-\delta^{2}} \frac{\left|\mathcal{D}_{3, \mathrm{DH}}^{(1)}(y)\right|}{\sqrt{1+y^{2}}} e^{i \Psi_{3, \mathrm{DH}}^{(1)}(y)-i \arctan (y)}, } \\
& \lim _{y \gg 1}\left|\mathcal{D}_{3, \mathrm{DH}}^{(1)}(y)\right| e^{i \Psi_{3, \mathrm{DH}}^{(1)}(y)}=\frac{1}{2}, \quad(\mathrm{~A} 18)
\end{aligned}
$$

where the superscript TM stands for toy model. By using Eqs. (A17) and (A18) we obtain

$$
\begin{aligned}
\lim _{y \gg 1}\left[\frac{\left|X_{3, \mathrm{DH}}^{(1, \mathrm{TM})}\right| e^{-i \delta_{3, \mathrm{DH}}^{(1, \mathrm{TM})}}}{\left.\left|X_{3, \mathrm{DH}}^{(1, \mathrm{BM})}\right| e^{-i \delta_{3, \mathrm{DH}}^{(1, \mathrm{BM})}}\right]}\right. & =\frac{9}{40}\left[\frac{c_{\mathrm{DH}} a^{3}}{k_{B}}\right] \frac{N_{\mathrm{corr}}}{\left(1-\delta^{2}\right)\left|T \chi_{T}\right|} \\
& \left.\simeq 2.2 \frac{N_{\mathrm{corr}}}{\left(1-\delta^{2}\right)\left|T \chi_{T}\right|}, \quad, \mathrm{A} 19\right)
\end{aligned}
$$

where the last approximate equality was obtained by using the values for glycerol $c_{\mathrm{DH}} \simeq 1.2 \times 10^{6} \mathrm{~J} / \mathrm{K} \mathrm{m}^{3}$ and $a^{3} \simeq$ $1.2 \times 10^{-28} \mathrm{~m}^{3}$. We thus have shown that, provided $N_{\text {corr }}$ is proportional to $\left|T \chi_{T}\right|$, the predictions of the box and toy model for $X_{3}^{(1)}\left(f \gg f_{\alpha}\right)$ are similar in phase and in magnitude. This is important since this corresponds to the observable and to the frequency range where the box model has been able to fit the experimental data. We end with two remarks.

(i) In glycerol around $204 \mathrm{~K}$ one finds $\left|T \chi_{T}\right| \simeq 10^{2}$ and in Ref. [33] a good fit of the measured $X_{3}^{(1)}$ was obtained within the toy model with $N_{\text {corr }}=15$ and $\delta=0.6$. Using these values in Eq. (A19) yields $X_{3}^{(3), \mathrm{TM}} / X_{3}^{(3), \mathrm{BM}} \simeq 0.5$, i.e., a value that is two times smaller than expected. This shows that the limit $y \gg 1$ is not precise enough to give the exact prefactors. Similarly, the phase of the measured $X_{3}^{(1)}\left(f \gg f_{\alpha}\right)$ is not $-\pi / 2$ but a factor of 2 smaller. We note furthermore that the exact value of $c_{\mathrm{DH}}$ chosen to fit the $X_{3}^{(1)}$ data within the box model depends on the material and that an adjustable factor, between 0.5 and 1, has been used in Ref. [52]. This adjustable factor is within the range of numerical uncertainty produced by our method using the limit $y \gg 1$.

(ii) Also applying this reasoning to $X_{3}^{(3)}$ does not yield a corresponding result since $\left|X_{3}^{(3), \mathrm{TM}} / X_{3}^{(3), \mathrm{BM}}\right| \propto y$, i.e., the two models never yield the same functional dependence on $y$ for the third-harmonic cubic susceptibility.
[1] L. Berthier and G. Biroli, Rev. Mod. Phys. 83, 587 (2011).

[2] G. Adam and J. H. Gibbs, J. Chem. Phys. 43, 139 (1965).

[3] W. Gotze and L. Sjogren, Rep. Prog. Phys. 55, 241 (1992).

[4] G. Biroli and J.-P. Bouchaud, Europhys. Lett. 67, 21 (2004).

[5] S. Franz and G. Parisi, J. Phys.: Condens. Matter 12, 6335 (2000); C. Donati, S. Franz, S. C. Glotzer, and G. Parisi, J. Non-Cryst. Solids 307-310, 215 (2002).
[6] G. Biroli, J.-P. Bouchaud, K. Miyazaki, and D. R. Reichman, Phys. Rev. Lett. 97, 195701 (2006).

[7] M. Dzero, J. Schmalian, and P. G. Wolynes, in Structural Glasses and Supercooled Liquids: Theory, Experiment, and Applications, edited by P. G. Wolynes and V. Lubchenko (Wiley, New York, 2012), pp. 193-222; G. Biroli and J.-P. Bouchaud, ibid., pp. 31-114; arXiv:0912.2542v1.

[8] A. Montanari and G. Semerjian, J. Stat. Phys. 125, 23 (2006). 
[9] L. Berthier, G. Biroli, J.-P. Bouchaud, L. Cipelletti, and W. van Saarloos, Dynamical Heterogeneities in Glasses, Colloids, and Granular Media (Oxford University Press, Oxford, 2011).

[10] D. Chandler and J. P. Garrahan, Annu. Rev. Phys. Chem. 61, 191 (2010).

[11] J.-P. Bouchaud and G. Biroli, Phys. Rev. B 72, 064204 (2005).

[12] C. Crauste-Thibierge, C. Brun, F. Ladieu, D. L'Hôte, G. Biroli, and J.-P. Bouchaud, Phys. Rev. Lett. 104, 165703 (2010).

[13] T. Bauer, P. Lunkenheimer, and A. Loidl, Phys. Rev. Lett. 111, 225702 (2013).

[14] M. Michl, Th. Bauer, P. Lunkenheimer, and A. Loidl, J. Chem. Phys. 144, 114506 (2016).

[15] R. Casalini, D. Fragiadakis, and C. M. Roland, J. Chem. Phys. 142, 064504 (2015).

[16] L. Berthier, G. Biroli, J.-P. Bouchaud, L. Cipeletti, D. El Masri, D. L'Hôte, F. Ladieu, and M. Pierno, Science 310, 1797 (2005).

[17] C. Dalle-Ferrier, C. Thibierge, C. Alba-Simionesco, L. Berthier, G. Biroli, J.-P. Bouchaud, F. Ladieu, D. L'Hôte, and G. Tarjus, Phys. Rev. E 76, 041510 (2007); L. Berthier, G. Biroli, J.-P. Bouchaud, W. Kob, K. Miyazaki, and D. R. Reichman, J. Chem. Phys. 126, 184503 (2007).

[18] S. Albert, Th. Bauer, M. Michl, G. Biroli, J.-P. Bouchaud, A. Loidl, P. Lunkenheimer, R. Tourbot, C. Wiertel-Gasquet, and F. Ladieu, Science 352, 1308 (2016).

[19] J. M. Brader, M. Siebenbürger, M. Ballauff, K. Reinheimer, M. Wilhelm, S. J. Frey, F. Weysser, and M. Fuchs, Phys. Rev. E 82, 061401 (2010).

[20] R. Seyboldt, D. Merger, F. Coupette, M. Siebenbürger, M. Ballauff, M. Wilhelm, and M. Fuchs, Soft Matter 12, 8825 (2016).

[21] M. Tarzia, G. Biroli, A. Lefèvre, and J.-P. Bouchaud, J. Chem. Phys. 132, 054501 (2010).

[22] R. Richert, J. Chem. Phys. 144, 114501 (2016).

[23] P. Kim, A. R. Young-Gonzales, and R. Richert, J. Chem. Phys. 145, 064510 (2016).

[24] C. Brun, F. Ladieu, D. L’Hôte, M. Tarzia, G. Biroli, and J.-P. Bouchaud, Phys. Rev. B 84, 104204 (2011).

[25] C. Brun, C. Crauste-Thibierge, F. Ladieu, and D. L'Hôte, J. Chem. Phys. 133, 234901 (2010).

[26] See Supplemental Material of Th. Bauer, P. Lunkenheimer, S. Kastner, and A. Loidl, Phys. Rev. Lett. 110, 107603 (2013).

[27] D. L'Hôte, R. Tourbot, F. Ladieu, and P. Gadige, Phys. Rev. B 90, 104202 (2014).

[28] For glycerol and propylene carbonate the plateau region can be modeled $[18,24]$, up to a multiplicative factor of order 1 , by the dielectric cubic response of a gas of noninteracting dipoles studied first by W. T. Coffey and B. V. Paranjape, Proc. R. Irish Acad. A 78, 17 (1978) and then by J. L. Déjardin and Y. P. Kalmykov, Phys. Rev. E 61, 1211 (2000). For the plastic crystal studied in Ref. [14], this is no longer the case owing to peculiar physics taking place at the molecular scale.

[29] As shown in the Appendix, within the standard RFOT where $\Psi \equiv \theta \equiv d / 2$, the barrier height is proportional to $\left(\ell^{\star} / a\right)^{d / 2}$, yielding a much slower dependence than the $\left(\ell^{\star} / a\right)^{d}$ dependence assumed in the Adam-Gibbs theory. One could naively think that such a large difference can be easily discriminated experimentally by comparing the $N_{\text {corr }}(T)$, drawn from $\chi_{3}(T)$ measurements, and $\chi_{T} \equiv\left|\partial \ln \left(\tau_{\alpha}\right) / \partial T\right|$. This is not the case since by adopting the Adam-Gibbs theory, one predicts $N_{\text {corr }} \propto$ $T^{2} \chi_{T}=\left|\partial \ln \left(\tau_{\alpha}\right) / \partial[1 / T]\right|$; however, this is also the case if the usual form $S_{c}(T)=S_{\infty}\left(1-T_{K} / T\right)$ is inserted in the RFOT, i.e., in Eq. (A11) with the recommended values $\Psi=\theta=d / 2$. Distinguishing between the Adam-Gibbs and RFOT theories thus requires more than the study of $N_{\text {corr }}(T)$. This is not so surprising since both theories agree on a key point, namely, the existence of a domain length $\ell^{\star}$ that grows upon cooling.

[30] This comes from the nonzero value of the configurational entropy in supercooled liquids. This configurational entropy does not exist in the case of spin glasses, hence there is a finite probability for an amorphously ordered domain to return to a given configuration even after having relaxed. As a result, instead of a hump for $X_{3}$ around $f_{\alpha}$, there is a plateau for $X_{3}\left(f \leqslant f_{\alpha}\right) \simeq X_{3}(0)$, the amplitude of which diverges when approaching the spin glass transition; see L. Lévy, Phys. Rev. B 38, 4963 (1988).

[31] It would be worth developing further the MCT analysis of [21] to obtain detailed analytical predictions of the phases.

[32] The growth of $\chi_{3}$ and $\chi_{5}$ can be induced by purely dynamical correlation, as it is the case in MCT theory and in the MCT regime. However, in the activated dynamics regime, the only theories that can explain a growth of $\chi_{3}$ and $\chi_{5}$ are the ones in which dynamical correlations are driven by static (point-to-set) correlations.

[33] F. Ladieu, C. Brun, and D. L'Hôte, Phys. Rev. B 85, 184207 (2012).

[34] U. Buchenau, Phys. Rev. E 93, 032608 (2016).

[35] U. Buchenau, J. Chem. Phys. 146, 214503 (2017).

[36] G. Diezemann, Phys. Rev. E 85, 051502 (2012).

[37] G. Diezemann, Phys. Rev. E 96, 022150 (2017).

[38] G. P. Johari, J. Chem. Phys. 138, 154503 (2013).

[39] G. P. Johari, J. Chem. Phys. 145, 164502 (2016).

[40] R. Richert and C. A. Angell, J. Chem. Phys. 108, 9016 (1998).

[41] G. Tarjus, S. A. Kivelson, Z. Nussinov, and P. Viot, J. Phys.: Condens. Matter 17, R1143 (2005).

[42] J.-P. Bouchaud and G. Biroli, J. Chem. Phys. 121, 7347 (2004).

[43] C. Cammarota, A. Cavagna, G. Gradenigo, T. S. Grigera, and P. Verrocchio, J. Chem. Phys. 131, 194901 (2009).

[44] The minimal value of $q$ is obtained with $\Psi=0$ and $\theta=d-1$, while the maximal value of $q$ comes from $\Psi=d$ and $\theta=d / 2$. For $d=3$ this yields $1 / 3 \leqslant q \leqslant 3 / 2$.

[45] S. Samanta and R. Richert, J. Chem. Phys. 142, 044504 (2015).

[46] A. R. Young-Gonzales, S. Samanta, and R. Richert, J. Chem. Phys. 143, 104504 (2015).

[47] B. Riechers, K. Samwer, and R. Richert, J. Chem. Phys. 142, 154504 (2015).

[48] S. Samanta, O. Yamamuro, and R. Richert, Thermochim. Acta 636, 57 (2016).

[49] B. Schiener, R. Böhmer, A. Loidl, and R. V. Chamberlin, Science 274, 752 (1996); B. Schiener, R. V. Chamberlin, G. Diezemann, and R. Böhmer, J. Chem. Phys. 107, 7746 (1997).

[50] R. Richert and S. Weinstein, Phys. Rev. Lett. 97, 095703 (2006).

[51] S. Weinstein and R. Richert, Phys. Rev. B 75, 064302 (2007).

[52] L.-M. Wang and R. Richert, Phys. Rev. Lett. 99, 185701 (2007).

[53] A. Khalife, U. Pathak, and R. Richert, Eur. Phys. J. B 83, 429 (2011).

[54] The only sound physical interpretation we can find is that this heat is related to the part of the entropy, the configurational entropy, that is gained once the system has time to relax and sample all possible metastable states. This is naturally encoded in the RFOT and is associated with the growth of $N_{\text {corr }}$. 
[55] C. Brun, C. Crauste-Thibierge, F. Ladieu, and D. L'Hôte, J. Chem. Phys. 134, 194507 (2011).

[56] J. D. Stevenson, J. Schmalian, and P. G. Wolynes, Nat. Phys. 2, 268 (2006).

[57] S. Samanta and R. Richert, J. Phys. Chem. B 120, 7737 (2016).

[58] C. M. Newman and D. S. Stein, J. Stat. Phys. 106, 213 (2002).

[59] D. S. Fisher, in Slow Relaxations and Non-equilibrium Dynamics in Condensed Matter, edited by J.-L. Barrat, M. V. Feigelman, J. Kurchan, and J. Dalibard, Proceedings of the Les Houches Summer School of Theoretical Physics, LXXVII (Springer, Berlin, 2003), Vol. 77, p. 534.

[60] The Adam-Gibbs theory assumes that the barrier height $\Delta$ for a system to relax is proportional to $N_{\text {corr }}$ and given by $\Delta_{0}(\ell / a)^{d}$, with $\Delta_{0}$ a constant. By using $\ln \left(\tau_{\alpha} / \tau_{0}\right)=\Delta / k_{B} T=$ $(\ell / a)^{d} \Delta_{0} / k_{B} T$, one understands that the relaxation processes will be overwhelmingly dominated by the fastest ones, i.e., by those corresponding to the smallest possible value of $\ell$. This value is obtained by replacing the inequality by an equality in Eq. (A6), which yields $(\ell / a)^{d}=k_{B} \ln 2 / S_{c}$ and finally $\ln \left(\tau_{\alpha} / \tau_{0}\right)=\ln (2) \Delta_{0} / T S_{c}$, which is similar to Eq. (9), up to a redefinition of $\Delta_{0}$ to absorb the factor $\ln 2$.

[61] This can be understood by considering an Ising model, of coupling constant $J$, with a domain containing $(\ell / a)^{d}$ up spins in a sea of down spins: The energy cost to split this domain in two is proportional to $J(\ell / a)^{(d-1)}$, the cost of splitting the two new domains of size $\ell / 2$ is proportional to $J[(\ell / 2) / a]^{(d-1)}$, and so on until all the spins have been flipped down. Summing up all these energy costs leads to a full energy cost of domain flipping, which goes as $(\ell / a)^{(d-1)}$. 